\title{
Taxonomy update for the family Alphasatellitidae: new subfamily, genera, and species
}

\author{
Arvind Varsani ${ }^{1,2}\left({ }^{10} \cdot\right.$ Darren P. Martin $^{3} \cdot$ John W. Randles $^{4} \cdot$ H. Josef Vetten ${ }^{5} \cdot$ John E. Thomas $^{6} \cdot$ Elvira Fiallo-Olivé $^{7}$. \\ Jesús Navas-Castillo ${ }^{7}$ Jean-Michel Lett ${ }^{8} \cdot$ F. Murilo Zerbini $^{9,10} \cdot$ Philippe Roumagnac $^{11,12} \cdot$ Bruno Gronenborn $^{13}$
}

Published online: 22 September 2021

(C) The Author(s), under exclusive licence to Springer-Verlag GmbH Austria, part of Springer Nature 2021

\begin{abstract}
Alphasatellites (family Alphasatellitidae) are circular, single-stranded DNA molecules ( 1-1.4 kb) that encode a replicationassociated protein and have commonly been associated with some members of the families Geminiviridae, Nanoviridae, and Metaxyviridae (recently established). Here, we provide a taxonomy update for the family Alphasatellitidae following the International Committee on Taxonomy of Viruses (ICTV) Ratification Vote held in March 2021. The taxonomic update includes the establishment of the new subfamily Petromoalphasatellitinae. This new subfamily includes three new genera as well as the genus Babusatellite, which previously belonged to the subfamily Nanoalphasatellitinae. Additionally, three new genera and 14 new species have been established in the subfamily Geminialphasatellitinae, as well as five new species in the subfamily Nanoalphasatellitinae.
\end{abstract}

\section{Introduction}

Alphasatellitidae is a family of replication-associated protein (Rep)-expressing circular, single-stranded DNA molecules $(\sim 1-1.4 \mathrm{~kb})$ that have commonly been found in association with some members of the families Geminiviridae (viruses in the genera Begomovirus and Mastrevirus) [14] and

Handling Editor: Sead Sabanadzovic.

Arvind Varsani

arvind.varsani@asu.edu

Bruno Gronenborn

bgronenborn@gmail.com

1 The Biodesign Center for Fundamental and Applied Microbiomics, Center for Evolution and Medicine, School of Life Sciences, Arizona State University, $1001 \mathrm{~S}$. McAllister Ave, Tempe, AZ 85287-5001, USA

2 Structural Biology Research Unit, Department of Integrative Biomedical Sciences, University of Cape Town, Cape Town 7925, South Africa

3 Computational Biology Division, Department of Integrative Biomedical Sciences, Institute of Infectious Diseases and Molecular Medicine, University of Cape Town Observatory, Cape Town 7925, South Africa

4 School of Agriculture Food and Wine, University of Adelaide, Adelaide, South Australia 5005, Australia

5 Im Spargelfeld 1, 38162 Cremlingen, Germany
Nanoviridae [12]. Similar molecules have also been found associated with coconut foliar decay virus [4] (family Metaxyviridae, genus Cofodevirus) [13]. Alphasatellites are unable to trans-replicate the bona fide genome components of these 'helper viruses' or vice versa, but they do rely on them for encapsidation, movement, and vector transmission [1].

Since the establishment of the family Alphasatellitidae in 2018 [1], various new alphasatellites have been identified,

6 Queensland Alliance for Agriculture and Food Innovation, The University of Queensland, GPO Box 267, Brisbane, Queensland 4001, Australia

7 Instituto de Hortofruticultura Subtropical y Mediterránea "La Mayora", Consejo Superior de Investigaciones Científicas-Universidad de Málaga (IHSM-CSIC-UMA), 29750 Algarrobo-Costa, Málaga, Spain

8 CIRAD, UMR PVBMT, Pôle de Protection des Plantes, 97410 Saint-Pierre, Ile de la Reunion, France

9 Departmento de Fitopatologia/BIOAGRO, Universidade Federal de Viçosa, Viçosa, MG 36570-900, Brazil

10 National Research Institute for Plant-Pest Interactions, Universidade Federal de Viçosa, Viçosa, MG 36570-900, Brazil

11 CIRAD, UMR PHIM, 34090 Montpellier, France

12 PHIM Plant Health Institute, Univ Montpellier, CIRAD, INRAE, Institut Agro, IRD, 34090 Montpellier, France

13 Im Broich 38, 50128 Bergheim-Fliesteden, Germany 
and here, we provide a taxonomic update. The update includes: (1) the establishment of a new subfamily, Petromoalphasatellitinae; (2) reassignment of existing family members and assignment of new members to the subfamily Petromoalphasatellitinae; (3) the establishment of three new genera and 14 new species in the subfamily Geminialphasatellitinae; and (4) the establishment of five new species in the subfamily Nanoalphasatellitinae.

\section{Geminialphasatellitinae}

A genus demarcation threshold of $70 \%$ and a species demarcation threshold of $88 \%$ based on genome-wide pairwise identity values were recommended by Briddon et al. [1] (Fig. 1). Accordingly, the members were classified into four genera (Ageyesisatellite, Clecrusatellite, Colecusatellite, and Gosmusatellite) and a total of 41 species. In the last couple of years, various geminialphasatellites have been identified, and using the taxonomic guidelines outlined by Briddon et al. [1], we established three new genera (Draflysatellite, Somasatellite, and Whiflysatellite) to accommodate the two previously unassigned species (Dragonfly associated alphasatellite and Whitefly associated Guatemala alphasatellite 1) and one new species (Sorghum mastrevirus associated alphasatellite). In addition to these, we established 12 new species in the genera Clecrusatellite $(n=7)$, Colecusatellite $(n=2)$, and Gosmusatellite $(n=3)$. The details of the new taxa are provided in Table 1. With all the new geminialphasatellites being identified, we reanalysed the distribution of pairwise identity values for 960 complete sequences with intact Rep open reading frames using SDT v1.2 [7] and determined that the $88 \%$ species demarcation threshold is still a valid criterion for their classification (Fig. 1).

\section{Nanoalphasatellitinae}

A genus demarcation threshold of $67 \%$ and a species demarcation threshold of $80 \%$ based on genome-wide pairwise identity values were recommended previously by Briddon et al. [1]. This resulted in the original creation of 19 species, which were assigned to seven genera (Babusatellite, Clostunsatellite, Fabenesatellite, Milvetsatellite, Mivedwarsatellite, Sophoyesatellite, and Subclovsatellite). The major change in this subfamily is the reassignment of the genus Babusatellite to the subfamily Petromoalphasatellitinae. We also established five new species in the genera Mivedwarsatellite $(n=3)$, Sophoyesatellite $(n=1)$, and Subclovsatellite $(n=1)$. To verify that, with the reassignment of Babusatellite to the subfamily Petromoalphasatellitinae, the species demarcation threshold of $80 \%$ is still valid, we reanalysed the distribution of pairwise identity values for 104 complete nanoalphasatellite sequences with intact Rep open reading frames using SDT v1.2 [7] and confirmed the species demarcation threshold (Fig. 1).

\section{Petromoalphasatellitinae}

In 2018, novel types of single-stranded DNA molecules comprising the genome of coconut foliar decay virus [4] (family Metaxyviridae) were identified. The virus was found to affect only coconut palm (Cocos nucifera) and is restricted to the Vanuatu Archipelago [8, 9]. Nine different alphasatellites are associated with coconut foliar decay virus. Our analysis of these alphasatellites revealed that, based on a species demarcation threshold of $81 \%$ sequence identity, they can be classified in several distinct species (Fig. 1). Applying a genus demarcation threshold of $68 \%$ sequence identity among members, three new genera, Cocosatellite (Coco for coconut), Coprasatellite (Copra for coconut meat), and Kobbarisatellite (Kobbari is an Indian dish from coconut meat) were established. Five of the seven alphasatellite species were assigned to the genus Cocosatellite, one to the genus Coprasatellite, and one to the genus Kobbarisatellite (Table 1).

In addition to the creation of these three genera, we reassigned the genus Babusatellite to the new subfamily Petromoalphasatellitinae, as these alphasatellites collectively associate with viruses that infect perennial tropical monocotyledonous plants. Moreover, their respective size range is between those of the members of the subfamilies Geminialphasatellitinae and Nanoalphasatellitinae. Finally, phylogenetic analysis clearly supports inclusion of the species of the genus Babusatellite in the same subfamily along with the species of the newly established genera Cocosatellite, Coprasatellite, and Kobbarisatellite (Fig. 2). The distribution of the pairwise identity values for the 28 petromoalphasatellites is shown in Fig. 1.

Based on the phylogenetic analysis coupled with pairwise comparisons, we moved three species (Banana bunchy top alphasatellite 2, Banana bunchy top alphasatellite 3, and Cardamom bushy dwarf alphasatellite) from the genus Babusatellite to a new genus, Muscarsatellite (Muscar from Musa and Elettaria cardamomum). A summary of the genera and species is provided in Table 1.

For the classification of new petromoalphasatellites, we recommend similar steps to those outlined by Briddon et al. [1]. To resolve conflict in cases where (1) a complete petromoalphasatellite sequence shares $\geq 81 \%$ pairwise identity with sequences of members of two different species or (2) a complete petromoalphasatellite sequence shares $\geq 81 \%$ pairwise identity with sequences of one or more members of a particular species while sharing $<81 \%$ identity with the sequences of the majority of the members of that particular species: 

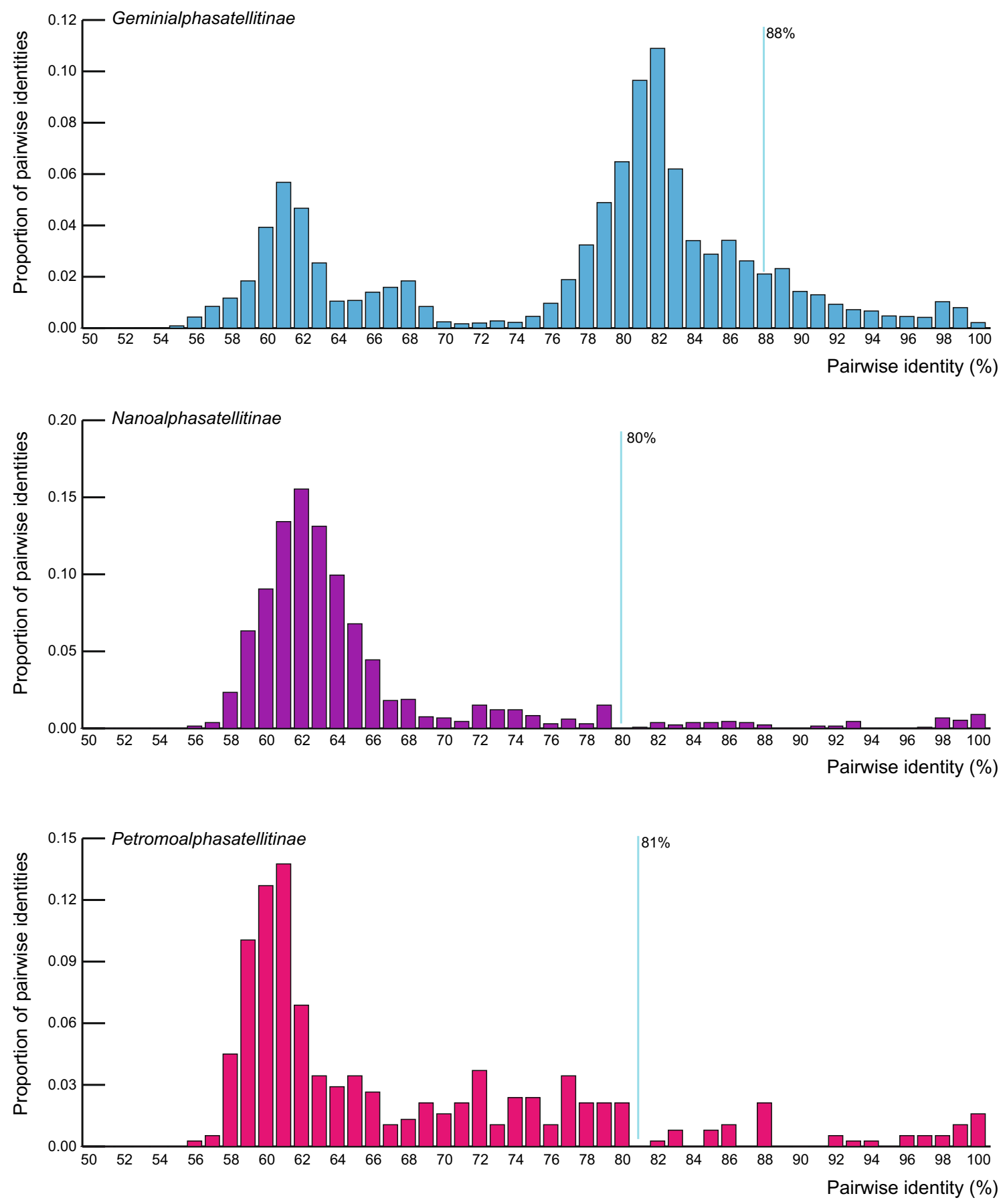

Fig. 1 Distribution of pairwise identity values of complete sequences with intact Rep ORFs of members of the subfamilies Geminialphasatellitinae $(n=960)$, Nanoalphasatellitinae $(n=104)$, and Petromoalphasatellitinae $(n=28)$, determined using SDT v1.2 [7]

(1) The new petromoalphasatellite should be assigned to the species whose members share with it the highest pairwise sequence identity.

(2) The petromoalphasatellite should be classified as belonging to any species in which it shares $\geq 81 \%$ pair- wise sequence identity with any existing member of that species, even if it has $<81 \%$ pairwise sequence identity to other members of that species. 
Table 1 Summary of the genera and species in the subfamilies Geminialphasatellitinae, Nanoalphasatellitinae, and Petromoalphasatellitinae

\begin{tabular}{|c|c|c|c|c|c|c|}
\hline Subfamily & Genus & & Species & Alphasatellite name & Isolate & Accession no. \\
\hline \multirow[t]{22}{*}{$\begin{array}{l}\text { Geminialphasatel- } \\
\text { litinae }\end{array}$} & \multirow{2}{*}{\multicolumn{2}{|c|}{ Ageyesisatellite }} & $\begin{array}{l}\text { Ageratum yellow vein } \\
\text { Singapore alphas- } \\
\text { atellite }\end{array}$ & $\begin{array}{l}\text { ageratum yellow vein } \\
\text { Singapore alphas- } \\
\text { atellite }\end{array}$ & SG-98 & AJ416153 \\
\hline & & & $\begin{array}{l}\text { Cotton leaf curl Saudi } \\
\text { Arabia alphasatel- } \\
\text { lite }\end{array}$ & $\begin{array}{l}\text { cotton leaf curl Saudi } \\
\text { Arabia alphasatel- } \\
\text { lite }\end{array}$ & SA-Jazan-13 & HG530543 \\
\hline & \multirow[t]{15}{*}{ Clecrusatellite } & $\diamond$ & $\begin{array}{l}\text { Ash gourd yellow } \\
\text { vein mosaic alphas- } \\
\text { atellite }\end{array}$ & $\begin{array}{l}\text { ash gourd yellow } \\
\text { vein mosaic alphas- } \\
\text { atellite }\end{array}$ & IN-UdA-15 & KX363561 \\
\hline & & $\diamond$ & $\begin{array}{l}\text { Capsicum India } \\
\text { alphasatellite }\end{array}$ & $\begin{array}{l}\text { begomovirus-associ- } \\
\text { ated alphasatellite } \\
\text { sp. }\end{array}$ & IN-PJ-Cap-15 & KU923759 \\
\hline & & \multirow[t]{6}{*}{$\diamond$} & $\begin{array}{l}\text { Chiapas weed alph- } \\
\text { asatellite }\end{array}$ & $\begin{array}{l}\text { begomovirus-associ- } \\
\text { ated alphasatellite }\end{array}$ & $\begin{array}{l}\text { MX-UHAsV-1-CD- } \\
\text { W-2014 }\end{array}$ & MN203219 \\
\hline & & & $\begin{array}{l}\text { Cleome leaf crumple } \\
\text { alphasatellite }\end{array}$ & $\begin{array}{l}\text { cleome leaf crumple } \\
\text { alphasatellite }\end{array}$ & $\begin{array}{l}\text { BR-Mato Grosso do } \\
\text { Sul-07 }\end{array}$ & FN436007 \\
\hline & & & $\begin{array}{l}\text { Croton yellow vein } \\
\text { mosaic alphasatel- } \\
\text { lite }\end{array}$ & $\begin{array}{l}\text { croton yellow vein } \\
\text { mosaic alphasatel- } \\
\text { lite }\end{array}$ & $\begin{array}{l}\text { IN-Haryana-Acaly- } \\
\text { pha-07 }\end{array}$ & FN658711 \\
\hline & & & $\begin{array}{l}\text { Euphorbia yellow } \\
\text { mosaic alphasatel- } \\
\text { lite }\end{array}$ & $\begin{array}{l}\text { euphorbia yellow } \\
\text { mosaic alphasatel- } \\
\text { lite }\end{array}$ & $\begin{array}{l}\text { BR-Mato grosso do } \\
\text { sul-1-07 }\end{array}$ & FN436008 \\
\hline & & & $\begin{array}{l}\text { Melon chlorotic } \\
\text { mosaic alphasatel- } \\
\text { lite }\end{array}$ & $\begin{array}{l}\text { melon chlorotic } \\
\text { mosaic alphasatel- } \\
\text { lite }\end{array}$ & $\begin{array}{l}\text { VE-2009_02_04_0- } \\
09\end{array}$ & HM163578 \\
\hline & & & $\begin{array}{l}\text { Sida Cuba alphasat- } \\
\text { ellite }\end{array}$ & $\begin{array}{l}\text { sida Cuba alphasat- } \\
\text { ellite }\end{array}$ & CU-Trinidad-07 & HE806451 \\
\hline & & $\diamond$ & $\begin{array}{l}\text { Tomato leaf curl } \\
\text { Anand alphasatel- } \\
\text { lite }\end{array}$ & $\begin{array}{l}\text { tomato leaf curl } \\
\text { Anand alphasatel- } \\
\text { lite }\end{array}$ & IN-Anand-2016 & MH577036 \\
\hline & & $\diamond$ & $\begin{array}{l}\text { Tomato leaf curl New } \\
\text { Delhi alphasatellite }\end{array}$ & $\begin{array}{l}\text { tomato leaf curl New } \\
\text { Delhi alphasatellite }\end{array}$ & IN-VNS_SP4-Luf-15 & MH550542 \\
\hline & & \multirow[t]{2}{*}{$\diamond$} & $\begin{array}{l}\text { Tomato leaf curl } \\
\text { Virudhunagar } \\
\text { alphasatellite }\end{array}$ & $\begin{array}{l}\text { tomato leaf curl } \\
\text { Virudhunagar } \\
\text { alphasatellite }\end{array}$ & IN-sev-Mom-16 & KY848691 \\
\hline & & & $\begin{array}{l}\text { Tomato yellow spot } \\
\text { alphasatellite }\end{array}$ & $\begin{array}{l}\text { tomato yellow spot } \\
\text { alphasatellite }\end{array}$ & BR-Dou1095.1-11 & KX348228 \\
\hline & & \multirow[t]{3}{*}{$\diamond$} & $\begin{array}{l}\text { Tomato yellow spot } \\
\text { alphasatellite } 2\end{array}$ & $\begin{array}{l}\text { tomato yellow spot } \\
\text { alphasatellite } 2\end{array}$ & $\begin{array}{l}\text { AR-Jujuy-Yuto- } \\
\text { Leonurus417-2008 }\end{array}$ & MN518743 \\
\hline & & & $\begin{array}{l}\text { Whitefly associated } \\
\text { Guatemala alphas- } \\
\text { atellite } 2\end{array}$ & $\begin{array}{l}\text { whitefly associated } \\
\text { Puerto Rico alphas- } \\
\text { atellite } 2\end{array}$ & GT-GtTo2-1-10 & КT099170 \\
\hline & & & $\begin{array}{l}\text { Whitefly associated } \\
\text { Puerto Rico alphas- } \\
\text { atellite } 1\end{array}$ & $\begin{array}{l}\text { whitefly associated } \\
\text { Puerto Rico alphas- } \\
\text { atellite } 1\end{array}$ & PR-PR3-6-10 & KT099173 \\
\hline & \multirow[t]{5}{*}{ Colecusatellite } & & $\begin{array}{l}\text { Ageratum enation } \\
\text { alphasatellite }\end{array}$ & $\begin{array}{l}\text { ageratum enation } \\
\text { alphasatellite }\end{array}$ & IN-Luc-12 & JX913532 \\
\hline & & & $\begin{array}{l}\text { Ageratum yellow vein } \\
\text { alphasatellite }\end{array}$ & $\begin{array}{l}\text { ageratum yellow vein } \\
\text { alphasatellite }\end{array}$ & SG-98 & AJ238493 \\
\hline & & & $\begin{array}{l}\text { Ageratum yellow vein } \\
\text { China alphasatellite }\end{array}$ & $\begin{array}{l}\text { ageratum yellow vein } \\
\text { China alphasatellite }\end{array}$ & $\begin{array}{l}\text { PH-Davao-SN3-Syn- } \\
\text { edrella nodiflora-12 }\end{array}$ & KF785752 \\
\hline & & & $\begin{array}{l}\text { Ageratum yellow vein } \\
\text { India alphasatellite }\end{array}$ & $\begin{array}{l}\text { ageratum yellow vein } \\
\text { India alphasatellite }\end{array}$ & $\begin{array}{l}\text { IN-Luc-parthe- } \\
\text { nium-12 }\end{array}$ & JX570736 \\
\hline & & & $\begin{array}{l}\text { Bhendi yellow vein } \\
\text { alphasatellite }\end{array}$ & $\begin{array}{l}\text { bhendi yellow vein } \\
\text { alphasatellite }\end{array}$ & IN-Har-07 & FN658716 \\
\hline
\end{tabular}


Table 1 (continued)

\begin{tabular}{|c|c|c|c|c|c|c|}
\hline Subfamily & Genus & & Species & Alphasatellite name & Isolate & Accession no \\
\hline & & & $\begin{array}{l}\text { Cassava mosaic } \\
\text { Madagascar alph- } \\
\text { asatellite }\end{array}$ & $\begin{array}{l}\text { cassava mosaic } \\
\text { Madagascar alphas- } \\
\text { atellite }\end{array}$ & MG-Diana-635A1-11 & HE984148 \\
\hline & & & $\begin{array}{l}\text { Chilli leaf curl alph- } \\
\text { asatellite }\end{array}$ & $\begin{array}{l}\text { chilli leaf curl alphas- } \\
\text { atellite }\end{array}$ & IN-273-06 & KF471043 \\
\hline & & & $\begin{array}{l}\text { Cotton leaf curl } \\
\text { Egypt alphasatellite }\end{array}$ & $\begin{array}{l}\text { cotton leaf curl Egypt } \\
\text { alphasatellite }\end{array}$ & EG-SB45-95 & AJ512960 \\
\hline & & & $\begin{array}{l}\text { Cotton leaf curl } \\
\text { Gezira alphasatel- } \\
\text { lite }\end{array}$ & $\begin{array}{l}\text { cotton leaf curl } \\
\text { Gezira alphasatel- } \\
\text { lite }\end{array}$ & ML-Bamako-okra-06 & EU589450 \\
\hline & & & $\begin{array}{l}\text { Cotton leaf curl Luc- } \\
\text { know alphasatellite }\end{array}$ & $\begin{array}{l}\text { cotton leaf curl Luc- } \\
\text { know alphasatellite }\end{array}$ & IN-Luc-10 & HQ343234 \\
\hline & & & $\begin{array}{l}\text { Cotton leaf curl Mul- } \\
\text { tan alphasatellite }\end{array}$ & $\begin{array}{l}\text { cotton leaf curl Mul- } \\
\text { tan colecusatellite }\end{array}$ & PK-Fai01-98 & AJ132344 \\
\hline & & & $\begin{array}{l}\text { Gossypium darwinii } \\
\text { symptomless alph- } \\
\text { asatellite }\end{array}$ & $\begin{array}{l}\text { Gossypium darwinii } \\
\text { symptomless alph- } \\
\text { asatellite }\end{array}$ & PK-Mul-Dav7C-06 & EU384623 \\
\hline & & & $\begin{array}{l}\text { Malvastrum yellow } \\
\text { mosaic alphasatel- } \\
\text { lite }\end{array}$ & $\begin{array}{l}\text { malvastrum yellow } \\
\text { mosaic alphasatel- } \\
\text { lite }\end{array}$ & $\mathrm{CN}-\mathrm{Hn} 39$ & AM236765 \\
\hline & & & $\begin{array}{l}\text { Malvastrum yellow } \\
\text { mosaic Cameroon } \\
\text { alphasatellite }\end{array}$ & $\begin{array}{l}\text { malvastrum yellow } \\
\text { mosaic Cameroon } \\
\text { satellite }\end{array}$ & $\begin{array}{l}\text { CM-Mundemba- } \\
\text { UMU1D1-08 }\end{array}$ & FN675297 \\
\hline & & & $\begin{array}{l}\text { Pedilanthus leaf curl } \\
\text { alphasatellite }\end{array}$ & $\begin{array}{l}\text { pedilanthus leaf curl } \\
\text { alphasatellite }\end{array}$ & IN-carrot & KX168428 \\
\hline & & & $\begin{array}{l}\text { Sida leaf curl alphas- } \\
\text { atellite }\end{array}$ & $\begin{array}{l}\text { sida leaf curl alphas- } \\
\text { atellite }\end{array}$ & $\begin{array}{l}\text { PK-Lahore-Alcea } \\
\text { rosea-06 }\end{array}$ & FR772088 \\
\hline & & $\diamond$ & $\begin{array}{l}\text { Sida leaf curl alphas- } \\
\text { atellite } 2\end{array}$ & $\begin{array}{l}\text { sida leaf curl alphas- } \\
\text { atellite }\end{array}$ & $\begin{array}{l}\text { IN-Gandhinagar- } \\
\text { Sida-2016 }\end{array}$ & KX513861 \\
\hline & & & $\begin{array}{l}\text { Sida yellow vein Viet- } \\
\text { nam alphasatellite }\end{array}$ & $\begin{array}{l}\text { sida yellow vein } \\
\text { Vietnam alphasat- } \\
\text { ellite }\end{array}$ & VN-Han-05 & DQ641718 \\
\hline & & & $\begin{array}{l}\text { Sunflower leaf curl } \\
\text { Karnataka alphas- } \\
\text { atellite }\end{array}$ & $\begin{array}{l}\text { sunflower leaf curl } \\
\text { Karnataka alphas- } \\
\text { atellite }\end{array}$ & IN-tomato-11 & JX569789 \\
\hline & & & $\begin{array}{l}\text { Synedrella leaf curl } \\
\text { alphasatellite }\end{array}$ & $\begin{array}{l}\text { synedrella leaf curl } \\
\text { alphasatellite }\end{array}$ & IN-Por-synf_1-09 & KJ939346 \\
\hline & & & $\begin{array}{l}\text { Tobacco curly shoot } \\
\text { alphasatellite }\end{array}$ & $\begin{array}{l}\text { tobacco curly shoot } \\
\text { alphasatellite }\end{array}$ & $\begin{array}{l}\text { IN-WSF1-Helian- } \\
\text { thus-10 }\end{array}$ & HQ407396 \\
\hline & & & $\begin{array}{l}\text { Tomato leaf curl } \\
\text { Buea alphasatellite }\end{array}$ & $\begin{array}{l}\text { tomato leaf curl Buea } \\
\text { alphasatellite }\end{array}$ & $\begin{array}{l}\text { CM-Buea- } \\
\text { TOS2D1-07 }\end{array}$ & FN675299 \\
\hline & & & $\begin{array}{l}\text { Tomato leaf curl } \\
\text { Cameroon alphas- } \\
\text { atellite }\end{array}$ & $\begin{array}{l}\text { tomato leaf curl } \\
\text { Cameroon alphas- } \\
\text { atellite }\end{array}$ & $\begin{array}{l}\text { CM-Buea- } \\
\text { OMHD3-08 }\end{array}$ & FN675296 \\
\hline & & $\diamond$ & $\begin{array}{l}\text { Tomato leaf curl } \\
\text { Pakistan alphasat- } \\
\text { ellite }\end{array}$ & $\begin{array}{l}\text { tomato leaf curl } \\
\text { alphasatellite }\end{array}$ & PK-SZ_258-Gos-15 & KY420167 \\
\hline & & & $\begin{array}{l}\text { Tomato yellow leaf } \\
\text { curl China alphas- } \\
\text { atellite }\end{array}$ & $\begin{array}{l}\text { tomato yellow leaf } \\
\text { curl China alphas- } \\
\text { atellite }\end{array}$ & PK-Fai-09 & AM749493 \\
\hline & & & $\begin{array}{l}\text { Tomato yellow leaf } \\
\text { curl Thailand alph- } \\
\text { asatellite }\end{array}$ & $\begin{array}{l}\text { tomato yellow leaf } \\
\text { curl Thailand alph- } \\
\text { asatellite }\end{array}$ & TH-Y70-03 & AJ579359 \\
\hline & & & $\begin{array}{l}\text { Tomato yellow leaf } \\
\text { curl Yunnan alphas- } \\
\text { atellite }\end{array}$ & $\begin{array}{l}\text { tomato yellow leaf } \\
\text { curl Yunnan alphas- } \\
\text { atellite }\end{array}$ & CN-YN4368_69-14 & KX759649 \\
\hline
\end{tabular}


Table 1 (continued)

\begin{tabular}{|c|c|c|c|c|c|c|c|}
\hline Subfamily & & Genus & & Species & Alphasatellite name & Isolate & Accession no. \\
\hline & $\checkmark$ & Draflysatellite & $\diamond$ & $\begin{array}{l}\text { Dragonfly associated } \\
\text { alphasatellite }\end{array}$ & $\begin{array}{l}\text { dragonfly associated } \\
\text { alphasatellite }\end{array}$ & PR-09 & JX458742 \\
\hline & & Gosmusatellite & $\diamond$ & $\begin{array}{l}\text { Cotton leaf curl Cam- } \\
\text { eroon alphasatellite }\end{array}$ & $\begin{array}{l}\text { cotton leaf curl } \\
\text { Gezira alphasatel- } \\
\text { lite } 3\end{array}$ & $\begin{array}{r}\text { CM-OBKG- } \\
\text { Okra-2007 }\end{array}$ & MN614472 \\
\hline & & & $\diamond$ & $\begin{array}{l}\text { Eclipta yellow vein } \\
\text { alphasatellite }\end{array}$ & $\begin{array}{l}\text { eclipta yellow vein } \\
\text { alphasatellite }\end{array}$ & PK-AlYVA-S3-13 & KX938425 \\
\hline & & & & $\begin{array}{l}\text { Gossypium musteli- } \\
\text { num symptomless } \\
\text { alphasatellite }\end{array}$ & $\begin{array}{l}\text { Gossypium musteli- } \\
\text { num symptomless } \\
\text { alphasatellite }\end{array}$ & PK-Mul-2-06 & EU384656 \\
\hline & & & & $\begin{array}{l}\text { Hollyhock yellow vein } \\
\text { alphasatellite }\end{array}$ & $\begin{array}{l}\text { hollyhock yellow } \\
\text { vein alphasatellite }\end{array}$ & PK-Lahor-17_5-06 & FR772086 \\
\hline & & & & $\begin{array}{l}\text { Mesta yellow vein } \\
\text { mosaic alphasatel- } \\
\text { lite }\end{array}$ & $\begin{array}{l}\text { mesta yellow vein } \\
\text { mosaic alphasatel- } \\
\text { lite }\end{array}$ & $\begin{array}{l}\text { IN-Ludhiana- } \\
\text { Okra-10 }\end{array}$ & JX183090 \\
\hline & & & & $\begin{array}{l}\text { Okra enation leaf } \\
\text { curl alphasatellite }\end{array}$ & $\begin{array}{l}\text { okra enation leaf curl } \\
\text { alphasatellite }\end{array}$ & IN-Surat-11 & HF546575 \\
\hline & & & & $\begin{array}{l}\text { Okra yellow crinkle } \\
\text { Cameroon alphas- } \\
\text { atellite }\end{array}$ & $\begin{array}{l}\text { okra yellow crinkle } \\
\text { Cameroon alphas- } \\
\text { atellite }\end{array}$ & CM-Lys1sp3-08 & FN675285 \\
\hline & & & & $\begin{array}{l}\text { Vernonia yellow vein } \\
\text { Fujian alphasatel- } \\
\text { lite }\end{array}$ & $\begin{array}{l}\text { vernonia yellow vein } \\
\text { Fujian alphasatel- } \\
\text { lite }\end{array}$ & $\begin{array}{l}\text { IN-Vamban-black- } \\
\text { gram-12 }\end{array}$ & КС959931 \\
\hline & $>$ & Somasatellite & $\diamond$ & $\begin{array}{l}\text { Sorghum mastrevirus } \\
\text { associated alphas- } \\
\text { atellite }\end{array}$ & $\begin{array}{l}\text { sorghum mastrevirus } \\
\text { associated alphas- } \\
\text { atellite }\end{array}$ & $\begin{array}{l}\text { RE-Bassin } \\
\text { plat-Sorghum } \\
\text { arundinaceum- } \\
\text { RE180_a-2017 }\end{array}$ & MN901968 \\
\hline & 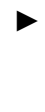 & Whiflysatellite & $\diamond$ & $\begin{array}{l}\text { Whitefly associated } \\
\text { Guatemala alphas- } \\
\text { atellite } 1\end{array}$ & $\begin{array}{l}\text { whitefly associated } \\
\text { Guatemala alphas- } \\
\text { atellite } 1\end{array}$ & $\begin{array}{l}\text { WfaGA-[GT- } \\
\text { GtTo2_2-12] }\end{array}$ & KT099172 \\
\hline \multirow[t]{11}{*}{$\begin{array}{l}\text { Nanoalphasatel- } \\
\text { litinae }\end{array}$} & & Clostunsatellite & & $\begin{array}{r}\text { Milk vetch dwarf } \\
\text { alphasatellite } 2\end{array}$ & $\begin{array}{r}\text { milk vetch dwarf } \\
\text { alphasatellite } 2\end{array}$ & MVDC3A-JR-C3-96 & AB000922 \\
\hline & & & & $\begin{array}{l}\text { Pea necrotic yellow } \\
\text { dwarf alphasatel- } \\
\text { lite } 2\end{array}$ & $\begin{array}{l}\text { pea necrotic yellow } \\
\text { dwarf alphasatel- } \\
\text { lite } 2\end{array}$ & $\begin{array}{l}\text { PNYD3A-AT-Gross_ } \\
\text { Enzersdorf_1-10 }\end{array}$ & КС979052 \\
\hline & & & & $\begin{array}{l}\text { Sophora yellow stunt } \\
\text { alphasatellite } 4\end{array}$ & $\begin{array}{l}\text { sophora yellow stunt } \\
\text { alphasatellite } 4\end{array}$ & $\begin{array}{l}\text { SYS9A-IR-Kerman- } \\
\text { Ta1-14 }\end{array}$ & KX534409 \\
\hline & & & & $\begin{array}{l}\text { Sophora yellow stunt } \\
\text { alphasatellite } 5\end{array}$ & $\begin{array}{l}\text { sophora yellow stunt } \\
\text { alphasatellite } 5\end{array}$ & $\begin{array}{l}\text { SYS8A-IR-Kerman- } \\
\text { Ta1-14 }\end{array}$ & KX534398 \\
\hline & & & & $\begin{array}{l}\text { Subterranean clover } \\
\text { stunt alphasatel- } \\
\text { lite } 2\end{array}$ & $\begin{array}{l}\text { subterranean clover } \\
\text { stunt alphasatel- } \\
\text { lite } 2\end{array}$ & $\begin{array}{l}\text { SCSC6A-[AU- } \\
\text { C6-93] }\end{array}$ & U16735 \\
\hline & & Fabenesatellite & & $\begin{array}{l}\text { Faba bean necrotic } \\
\text { yellows alphasatel- } \\
\text { lite } 2\end{array}$ & $\begin{array}{l}\text { faba bean necrotic } \\
\text { yellows alphasatel- } \\
\text { lite } 2\end{array}$ & $\begin{array}{l}\text { FBNYC9A-SY- } \\
\text { C9-93 }\end{array}$ & AJ005966 \\
\hline & & Milvetsatellite & & $\begin{array}{r}\text { Milk vetch dwarf } \\
\text { alphasatellite } 3\end{array}$ & $\begin{array}{l}\text { milk vetch dwarf } \\
\text { alphasatellite } 3\end{array}$ & $\begin{array}{l}\text { MVDC10A-JR- } \\
\text { C10-97 }\end{array}$ & AB009047 \\
\hline & & Mivedwarsatellite & & $\begin{array}{l}\text { Faba bean necrotic } \\
\text { stunt alphasatellite }\end{array}$ & $\begin{array}{l}\text { faba bean necrotic } \\
\text { stunt alphasatellite }\end{array}$ & AZ-12b-10 & КС978990 \\
\hline & & & & $\begin{array}{r}\text { Milk vetch dwarf } \\
\text { alphasatellite } 1\end{array}$ & $\begin{array}{r}\text { milk vetch dwarf } \\
\text { alphasatellite } 1\end{array}$ & MVDC1A-JR-C1-96 & AB000920 \\
\hline & & & $\diamond$ & $\begin{array}{l}\text { Milk vetch dwarf } \\
\text { China alphasatellite }\end{array}$ & $\begin{array}{l}\text { milk vetch dwarf } \mathrm{C} 1 \\
\text { alphasatellite }\end{array}$ & CN-G48-2017 & MN059431 \\
\hline & & & $\diamond$ & $\begin{array}{l}\text { Parsley severe stunt } \\
\text { alphasatellite } 3\end{array}$ & $\begin{array}{l}\text { parsley severe stunt } \\
\text { alphasatellite } 3\end{array}$ & DE-Pa21-2017 & MK039141 \\
\hline
\end{tabular}


Table 1 (continued)

\begin{tabular}{|c|c|c|c|c|c|c|c|}
\hline Subfamily & & Genus & & Species & Alphasatellite name & Isolate & Accession no. \\
\hline & & & $\diamond$ & $\begin{array}{l}\text { Parsley severe stunt } \\
\text { alphasatellite } 4\end{array}$ & $\begin{array}{l}\text { parsley severe stunt } \\
\text { alphasatellite } 4\end{array}$ & DE-Pa21-2017 & MK039142 \\
\hline & & & & $\begin{array}{l}\text { Pea necrotic yellow } \\
\text { dwarf alphasatel- } \\
\text { lite } 1\end{array}$ & $\begin{array}{l}\text { pea necrotic yellow } \\
\text { dwarf alphasatel- } \\
\text { lite } 1\end{array}$ & $\begin{array}{l}\text { PNYD1A-AT-Gross_ } \\
\text { Enzersdorf_1-10 }\end{array}$ & КС979051 \\
\hline & & & & $\begin{array}{l}\text { Sophora yellow stunt } \\
\text { alphasatellite } 2\end{array}$ & $\begin{array}{l}\text { sophora yellow stunt } \\
\text { alphasatellite } 2\end{array}$ & $\begin{array}{l}\text { SYS4A-IR-Kerman- } \\
\text { Ta1-14 }\end{array}$ & KX534408 \\
\hline & & Sophoyesatellite & $\diamond$ & $\begin{array}{l}\text { Cow vetch latent } \\
\text { alphasatellite }\end{array}$ & $\begin{array}{l}\text { cow vetch latent virus } \\
\text { alphasatellite }\end{array}$ & $\begin{array}{l}\text { FR-VcLV_Sam- } \\
\text { buc-10 }\end{array}$ & MF535455 \\
\hline & & & & $\begin{array}{l}\text { Sophora yellow stunt } \\
\text { alphasatellite } 3\end{array}$ & $\begin{array}{l}\text { sophora yellow stunt } \\
\text { alphasatellite } 3\end{array}$ & $\begin{array}{l}\text { SYS7A-IR-Kerman- } \\
\text { Ta1-14 }\end{array}$ & KX534400 \\
\hline & & Subclovsatellite & & $\begin{array}{l}\text { Faba bean necrotic } \\
\text { yellows alphasatel- } \\
\text { lite } 1\end{array}$ & $\begin{array}{l}\text { faba bean necrotic } \\
\text { yellows alphasatel- } \\
\text { lite } 1\end{array}$ & $\begin{array}{l}\text { FBNYC1A-SY- } \\
\text { C1-88 }\end{array}$ & X80879 \\
\hline & & & $\diamond$ & $\begin{array}{l}\text { Faba bean necrotic } \\
\text { yellows alphasatel- } \\
\text { lite } 3\end{array}$ & $\begin{array}{l}\text { faba bean necrotic } \\
\text { yellows virus asso- } \\
\text { ciated alphasatel- } \\
\text { lite } 1\end{array}$ & TN-Tuf9_1-15 & MF510471 \\
\hline & & & & $\begin{array}{l}\text { Sophora yellow stunt } \\
\text { alphasatellite } 1\end{array}$ & $\begin{array}{l}\text { sophora yellow stunt } \\
\text { alphasatellite } 1\end{array}$ & $\begin{array}{l}\text { SYSA1-[IR-Kerman- } \\
\text { Ta1-14] }\end{array}$ & KX534399 \\
\hline & & & & $\begin{array}{l}\text { Subterranean clover } \\
\text { stunt alphasatel- } \\
\text { lite } 1\end{array}$ & $\begin{array}{l}\text { subterranean clover } \\
\text { stunt alphasatel- } \\
\text { lite } 1\end{array}$ & SCSC2A-AU-C2-93 & U16731 \\
\hline $\begin{array}{l}\text { Petromoalphasatel- } \\
\text { litinae }\end{array}$ & & Babusatellite & & $\begin{array}{l}\text { Banana bunchy top } \\
\text { alphasatellite } 1\end{array}$ & $\begin{array}{l}\text { banana bunchy top } \\
\text { alphasatellite } 1\end{array}$ & BBTS2A-93 & L32167 \\
\hline & $>$ & Cocosatellite & $\diamond$ & $\begin{array}{l}\text { Coconut foliar decay } \\
\text { alphasatellite } 1\end{array}$ & $\begin{array}{l}\text { coconut foliar decay } \\
\text { alphasatellite } 1\end{array}$ & VU-85 & M29963 \\
\hline & & & $\diamond$ & $\begin{array}{l}\text { Coconut foliar decay } \\
\text { alphasatellite } 2\end{array}$ & $\begin{array}{l}\text { coconut foliar decay } \\
\text { alphasatellite } 2\end{array}$ & VU-89 & MF926426 \\
\hline & & & $\Delta$ & $\begin{array}{l}\text { Coconut foliar decay } \\
\text { alphasatellite } 4\end{array}$ & $\begin{array}{l}\text { coconut foliar decay } \\
\text { alphasatellite } 4\end{array}$ & VU-89 & MF926429 \\
\hline & & & $\diamond$ & $\begin{array}{l}\text { Coconut foliar decay } \\
\text { alphasatellite } 5\end{array}$ & $\begin{array}{l}\text { coconut foliar decay } \\
\text { alphasatellite } 5\end{array}$ & VU-89 & MF926430 \\
\hline & $>$ & Coprasatellite & $\diamond$ & $\begin{array}{l}\text { Coconut foliar decay } \\
\text { alphasatellite } 7\end{array}$ & $\begin{array}{l}\text { coconut foliar decay } \\
\text { alphasatellite } 7\end{array}$ & VU-89 & MF926432 \\
\hline & $>$ & Kobbarisatellite & $\diamond$ & $\begin{array}{l}\text { Coconut foliar decay } \\
\text { alphasatellite } 3\end{array}$ & $\begin{array}{l}\text { coconut foliar decay } \\
\text { alphasatellite } 3\end{array}$ & VU-89 & MF926427 \\
\hline & $>$ & Muscarsatellite & $\diamond$ & $\begin{array}{l}\text { Banana bunchy top } \\
\text { alphasatellite } 2\end{array}$ & $\begin{array}{l}\text { banana bunchy top } \\
\text { alphasatellite } 2\end{array}$ & $\begin{array}{l}\text { BBTS3A-VN-VB- } \\
\text { sat3-00 }\end{array}$ & AF416471 \\
\hline & & & $\diamond$ & $\begin{array}{l}\text { Banana bunchy top } \\
\text { alphasatellite } 3\end{array}$ & $\begin{array}{l}\text { banana bunchy top } \\
\text { alphasatellite } 3\end{array}$ & $\begin{array}{l}\text { BBTHA-CN-Hai- } \\
\text { kou-10 }\end{array}$ & HQ616080 \\
\hline & & & $\diamond$ & $\begin{array}{l}\text { Cardamom bushy } \\
\text { dwarf alphasatellite }\end{array}$ & $\begin{array}{l}\text { cardamom bushy } \\
\text { dwarf alphasatellite }\end{array}$ & IN-Kalimpong-07 & KF435148 \\
\hline
\end{tabular}

The symbol "@" indicates a new subfamily, the symbol "

\section{Concluding remarks}

As more sequence data become available, more changes to Alphasatellitidae taxonomy will certainly occur. We would also like to inform the Alphasatellitidae research community that a standardized binomial species nomenclature, consisting of the genus name and a free-form species epithet, has been ratified by the International
Committee on Taxonomy of Viruses (ICTV) [10]. This needs to be adopted for currently established species in the family Alphasatellitidae by the year 2023. Thus, we encourage the community to engage with the ICTV Geminiviridae and Tolecusatellitidae Study Group and the Nanoviridae Study Group to determine the binomial names for current and new species in the family Alphasatellitidae. 


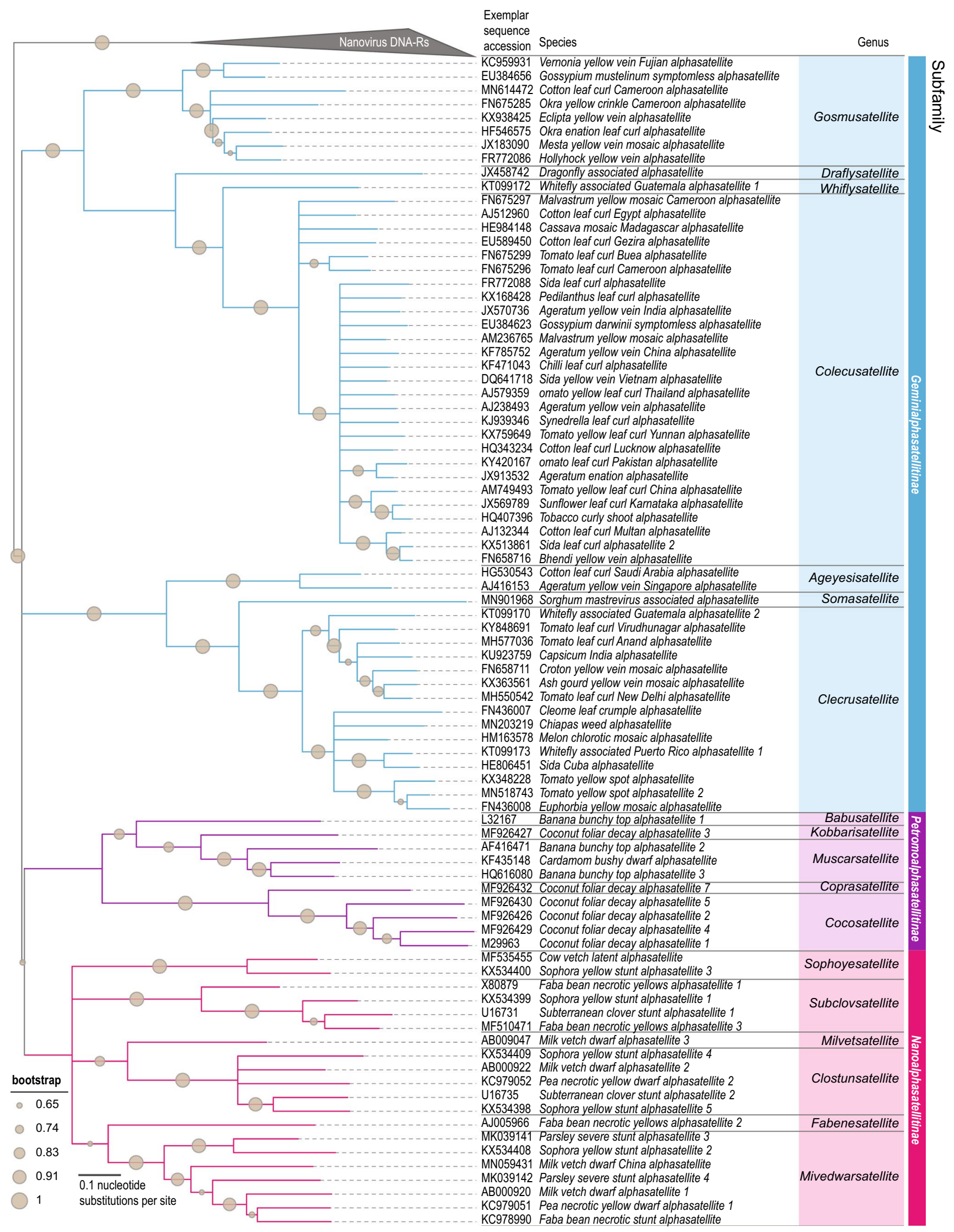


४Fig. 2 Maximum-likelihood phylogenetic tree constructed from a MUSCLE [3] sequence alignment of representative alphasatellites using PHYML [5] with the GTR+I+G4 nucleotide substitution model (determined to be the best-fitting model by jModelTest [2]). The tree shows support for the proposed establishment of the new subfamily Petromoalphasatellitinae as well as support for the reassignment of the viruses in the genus Babuvirus. Branches with less than $60 \%$ bootstrap support have been collapsed using TreeGraph2 [11]. The tree was visualized in iToL v6 [6].

Acknowledgment JML is supported by the European Union (ERDF), the Conseil Régional de La Réunion, and CIRAD.

\section{Declarations}

Conflict of interest The authors declare no conflicts of interest.

\section{References}

1. Briddon RW, Martin DP, Roumagnac P, Navas-Castillo J, FialloOlivé E, Moriones E, Lett JM, Zerbini FM, Varsani A (2018) Alphasatellitidae: a new family with two subfamilies for the classification of geminivirus- and nanovirus-associated alphasatellites. Arch Virol 163:2587-2600

2. Darriba D, Taboada GL, Doallo R, Posada D (2012) jModelTest 2: more models, new heuristics and parallel computing. Nat Methods 9:772

3. Edgar RC (2004) MUSCLE: multiple sequence alignment with high accuracy and high throughput. Nucleic Acids Res 32:1792-1797

4. Gronenborn B, Randles JW, Knierim D, Barriere Q, Vetten HJ, Warthmann N, Cornu D, Sileye T, Winter S, Timchenko T (2018) Analysis of DNAs associated with coconut foliar decay disease implicates a unique single-stranded DNA virus representing a new taxon. Sci Rep 8:5698

5. Guindon S, Dufayard JF, Lefort V, Anisimova M, Hordijk W, Gascuel O (2010) New algorithms and methods to estimate maximum-likelihood phylogenies: assessing the performance of PhyML 3.0. Syst Biol 59:307-321

6. Letunic I, Bork P (2021) Interactive Tree Of Life (iTOL) v5: an online tool for phylogenetic tree display and annotation. Nucleic Acids Res 49:W293-W296
7. Muhire BM, Varsani A, Martin DP (2014) SDT: a virus classification tool based on pairwise sequence alignment and identity calculation. PLoS One 9:e108277

8. Randles JW, Julia JF, Calvez C, Dollet M (1986) Association of single-stranded DNA with the foliar decay disease of coconut palm in Vanuatu. Phytopathology 76:889-894

9. Randles JW, Hanold D, Julia JF (1987) Small circular singlestranded DNA associated with foliar decay disease of coconut palm in Vanuatu. J Gen Virol 68:273-280

10. Siddell SG, Walker PJ, Lefkowitz EJ, Mushegian AR, Dutilh BE, Harrach B, Harrison RL, Junglen S, Knowles NJ, Kropinski AM, Krupovic M, Kuhn JH, Nibert ML, Rubino L, Sabanadzovic S, Simmonds P, Varsani A, Zerbini FM, Davison AJ (2020) Binomial nomenclature for virus species: a consultation. Arch Virol 165:519-525

11. Stover BC, Muller KF (2010) TreeGraph 2: combining and visualizing evidence from different phylogenetic analyses. BMC Bioinf 11:7

12. Thomas JE, Gronenborn B, Harding RM, Mandal B, Grigoras I, Randles JW, Sano Y, Timchenko T, Vetten HJ, Yeh HH, Ziebell H, ICTV Report Consortium (2021) ICTV virus taxonomy profile: Nanoviridae. J Gen Virol 102

13. Walker PJ, Siddell SG, Lefkowitz EJ, Mushegian AR, Adriaenssens EM, Alfenas-Zerbini P, Davison AJ, Dempsey DM, Dutilh BE, Garcia ML, Harrach B, Harrison RL, Hendrickson RC, Junglen S, Knowles NJ, Krupovic M, Kuhn JH, Lambert AJ, Lobocka M, Nibert ML, Oksanen HM, Orton RJ, Robertson DL, Rubino L, Sabanadzovic S, Simmonds P, Smith DB, Suzuki N, Van Dooerslaer K, Vandamme AM, Varsani A, Zerbini FM (2021) Changes to virus taxonomy and to the International Code of Virus Classification and Nomenclature ratified by the International Committee on Taxonomy of Viruses (2021). Arch Virol 166:2633-2648

14. Zerbini FM, Briddon RW, Idris A, Martin DP, Moriones E, NavasCastillo J, Rivera-Bustamante R, Roumagnac P, Varsani A, ICTV Report Consortium (2017) ICTV virus taxonomy profile: Geminiviridae. J Gen Virol 98:131-133

Publisher's Note Springer Nature remains neutral with regard to jurisdictional claims in published maps and institutional affiliations. 